\title{
Development and test of a decision aid for shared decision making in patients with anterior cruciate ligament injury
}

\author{
Hanne Mainz ${ }^{1}$, Lone Frandsen ${ }^{1}$, Martin Lind ${ }^{1}$, Peter Faun $\varnothing^{1}$, and Kirsten Lomborg ${ }^{2}$ \\ ${ }^{1}$ Aarhus University Hospital \\ ${ }^{2}$ Aarhus Universitet Institut for Klinisk Medicin
}

October 19, 2020

\begin{abstract}
Rationale, aims and objectives Patients with anterior crucial ligament injury are faced with a choice between surgery or non-surgical treatment with intensive rehabilitation. Evidence shows that surgical treatment is not superior to non-surgical treatment. To offer patients a treatment meeting their individual values, lifestyle and conditions, patients must be involved in the decision-making. The aim of the study was to develop and evaluate a patient decision aid to support shared decisionmaking. Method Development of the patient decision aid was based on international criteria, current literature, and former patients' experiences and suggestions on how to optimize the decision-making process. The patient decision aid was evaluated by the SDM-Q9 questionnaire and semi-structured interviews with patients and doctors. Results A patient decision aid for patients with and an anterior crucial ligament injury was developed. On a scale from 0-5, patients experienced a high degree of shared decision-making in their treatment decision both before (score 4.3) and after (score 4.3) implementation of the patient decision aid. No statically significant difference was found $(p=0.72)$. From interviews, patients expressed that they found the patient decision aid very useful. Especially, reflection time was important for some patients. Doctors reported that the patient decision aid improved shared decision-making by supporting the dialogue clarifying patients' values concerning issues important for treatment choices. Conclusion No statically significant difference in the SDM Q9 -score was found between patients' perceptions of shared decision-making before and after implementation of a patient decision aid. However, patients experienced the decision aid as very useful when making treatment decisions, and doctors reported that it improved the dialogue clarifying patients' values important for the treatment options. Keywords patient-centered care, evaluation, person-centered medicine
\end{abstract}

Title page

Title

Development and test of a decision aid for shared decision making in patients with anterior cruciate ligament injury

\section{Short running title}

Patient decision aid for shared decision making

\section{Authors}

Hanne Mainz ${ }^{1}$, RN, MHS, PhD, Clinical nurse specialist;

Lone Frandsen ${ }^{1}$, RN, DH, Specialist nurse;

Martin Lind ${ }^{1}, \mathrm{MD}, \mathrm{PhD}$ Professor,

Peter Fauno ${ }^{1}$, MD, Sector Chief in the Clinic of sports traumatology; 
Kirsten Lomborg ${ }^{2}, \mathrm{RN}, \mathrm{MSN}, \mathrm{PhD}$, Professor

${ }^{1}$ Clinic of Sports Traumatology, Department of Orthopaedic Surgery, Aarhus University Hospital, Denmark

2 Department of Clinical Medicine, Aarhus University, Denmark

Author information

Hanne Mainz, RN, MHS, PhD, Clinical nurse specialist; Clinic of Sports Traumatology, Department of Orthopaedic Surgery, Aarhus University Hospital, Denmark

Lone Frandsen, RN, DH, Specialist nurse; Clinic of Sports Traumatology, Department of Orthopaedic Surgery, Aarhus University Hospital, Denmark

Martin Lind, MD, PhD Professor, Clinic of Sports Traumatology, Department of Orthopaedic Surgery, Aarhus University Hospital, Denmark

Peter Fauno, MD, Sector Chief in the Clinic of sports traumatology; Clinic of Sports Traumatology, Department of Orthopaedic Surgery, Aarhus University Hospital, Denmark

Kirsten Lomborg, RN, MSN, PhD, Professor; Department of Clinical Medicine, Aarhus University, Denmark

\section{Corresponding author}

Hanne Mainz,

Department of Orthopaedic Surgery,

Aarhus University Hospital

Palle Juul-Jensens Boulevard 998200 Århus N. Denmark

email: hanmai@rm.dk

phone: +4526569767

\section{Acknowledgements}

We are grateful to all patients who participated in this study. We would also like to thank our colleagues in the Clinic of Sports Traumatology sports for their commitment and support in the project. Finally, we want to thank our colleagues at "Center for Research in Patient Involvement", Aarhus University Hospital, Denmark for good inspiration and encouragement.

\section{Conflict of interest}

No conflicts to declare

\section{Keywords}

patient-centered care, evaluation, person-centered medicine

\section{Funder}

Health Research Fund of Central Denmark Region and Department of Clinical Medicine, Aarhus University. Denmark

\section{Hosted file}

Main_document._Development_and_test_of_a_decision_aid_ACL.pdf available at https://authorea . com/users/368291/articles/487486-development-and-test-of-a-decision-aid-for-shareddecision-making-in-patients-with-anterior-cruciate-ligament-injury 


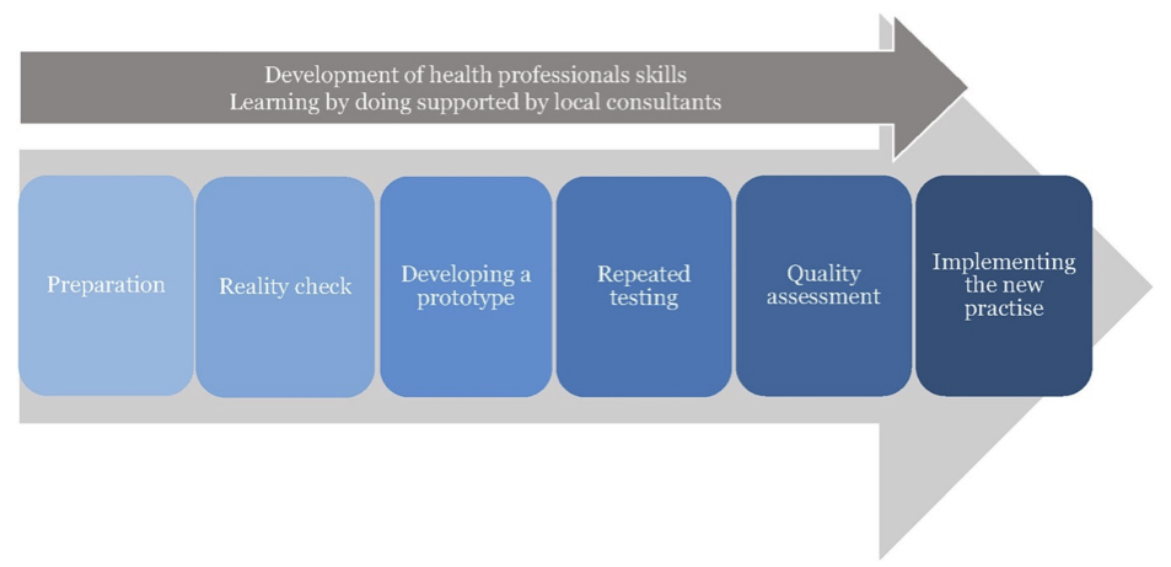

\section{Hosted file}

Tables. Development and test of a decision aid ACL.pdf available at https://authorea.com/ users/368291/articles/487486-development-and-test-of-a-decision-aid-for-shared-decisionmaking-in-patients-with-anterior-cruciate-ligament-injury 